EPJ Web of Conferences 60, 16014 (2013)

DOI: $10.1051 /$ epjconf $/ 20136016014$

(C) Owned by the authors, published by EDP Sciences, 2013

\title{
Measurements of the top-quark properties at CMS
}

\author{
Abideh Jafari for the CMS collaboration ${ }^{1,}$ a \\ ${ }^{1}$ Institute for Research in Fundamental Sciences (IPM)
}

\begin{abstract}
We present different measurements of the top quark properties in production and decay. The measurements are performed using the LHC proton-proton $(p p)$ collisions at $7 \mathrm{TeV}$ and $8 \mathrm{TeV}$ center-of-mass energies collected by the CMS detector during 2011 and 2012 runs, respectively. The study of production properties consists of charge asymmetry, $t \bar{t}$ spin correlation and the vector boson associated production of $t \overline{\text { }}$. The top quark properties in decay are studied via the measurement of the b-quark content, $R=\mathcal{B}(t \rightarrow W b) / \mathcal{B}(t \rightarrow W q)$, the top quark charge, the FCNC process of $t \rightarrow Z q$ and the W-helicity fractions. The results of the last measurement are used to set limits on the $t W b$ anomalous couplings. The W-helicity fractions are measured in the electroweakly produced (single-) top quarks, too.
\end{abstract}

\section{Introduction}

The top quark is the heaviest known particle with a mass close to the scale of the electroweak symmetry breaking. Its short lifetime, shorter than the hadronization and the spin decorrelation timescales, allows for the study of the bare quark properties via the decay products. The LHC [1] proton-proton collisions at $\sqrt{s}=7$ and $\sqrt{s}=$ $8 \mathrm{TeV}$ are collected by the CMS [2] detector during 2011 and 2012 runs, respectively. These datasets are used for the measurement of the top quark properties presented in this note.

\section{Charge Asymmetry}

The interference between the born approximation and the box diagram and between the initial and final state radiations (ISR/FSR) introduce a correlation between the direction of incoming and outgoing (anti-)quarks in $t \bar{t}$ production [3], [4]. Such correlation leads to a charge asymmetry, $A_{C}$, which is observable in the rapidity difference, $y_{t}-y_{\bar{t}}$. The CDF and D0 experiments have reported more than two standard deviation from the Standard Model (SM) expectation for this asymmetry [5], [6]. At the LHC, the charge asymmetry is investigated using the $\Delta|y|=\left|y_{t}\right|-\left|y_{t}\right|$ variable. The reason is that in $p p$ collisions, the anti-quarks contributing to quark-anti-quark annihilation are sea quarks with relatively smaller momentum fractions. Hence, the resulting top quarks are more boosted towards the forward regions. The CMS experiment has performed inclusive and differential measurements of $A_{C}$ at $\sqrt{s}=7 \mathrm{TeV}$ in lepton+jets final state [7]. The inclusive measurement results in $A_{C}=0.004 \pm 0.010$ (stat.) \pm 0.011 (syst.) which is consistent with the SM predictions

a. e-mail: Abideh.Jafari@ cern.ch at NLO, $A_{C}=0.0115 \pm 0.0006[8]$. Figure 1(a) illustrates the background subtracted and unfolded distribution of $A_{C}$ compared with the SM NLO calculations. The $t \bar{t}$ charge asymmetry can be influenced by changes in boundary conditions as well as by the physics beyond the SM. The variable $A_{C}$ is therefore measured differentially in those kinematic quantities which are sensitive to different aspects of charge asymmetry generation, namely the rapidity $\left(\left|y_{t \bar{t}}\right|\right)$, the transverse momentum $\left(p_{T, t \bar{t}}\right)$ and the invariant mass $\left(m_{t \bar{t}}\right)$ of the $t \bar{t}$ system. The charge asymmetry is expected to increase with $\left|y_{t \bar{t}}\right|$ due to the prominent $q \bar{q}$ annihilations in larger rapidities. The initial and final state radiations lead to on average higher transverse momenta of the $t \bar{t}$ system while they contribute negatively to $A_{C}$. The $q \bar{q}$ annihilation increase with $m_{t \bar{t}}$ hence one expects more pronounced asymmetry at higher $m_{t \bar{t}}$ values. Besides, new heavy particles may contribute in $t \bar{t}$ production, therefore interfere with the $m_{t \bar{t}}$ of charge asymmetry. In Figures $1(\mathrm{~b}), 1(\mathrm{c})$ and $1(\mathrm{~d})$ the differential $A_{C}$ measurements are found to be consistent with the SM NLO expectations. The measurements are however accompanied by large uncertainties. They are also compared with the predictions of an effective model with axial-vector coupling for gluon [9]. This model can describe the Tevatron $m_{t \bar{t}}$ dependence of the charge asymmetry. In dilepton final state at $\sqrt{s}=7 \mathrm{TeV}$, the charge asymmetry is found to be $A_{C}=0.05 \pm 0.04$ (stat.) ${ }_{-0.032}^{+0.010}$ (syst.) [10] which is in agreement with the SM NLO calculations, $0.0123 \pm 0.005$ [11]. Another observables investigated in this channel for the charge asymmetry is the difference between the leptons pseudorapidities, $\Delta\left|\eta_{l}\right|$. The measurement yields $A_{C}^{\Delta|\eta|}=0.010 \pm 0.015$ (stat.) \pm 0.006 (syst.) that agrees with SM expectations $0.0156 \pm 0.0007$ [11]. The top forward-backward asymmetry is also measured to be $A_{F B}^{\text {top }}=-0.010 \pm 0.034$ (stat.) \pm 0.026 (syst.), compatible with PowHeg [12] calculations, $0.005 \pm 0.0004$. 


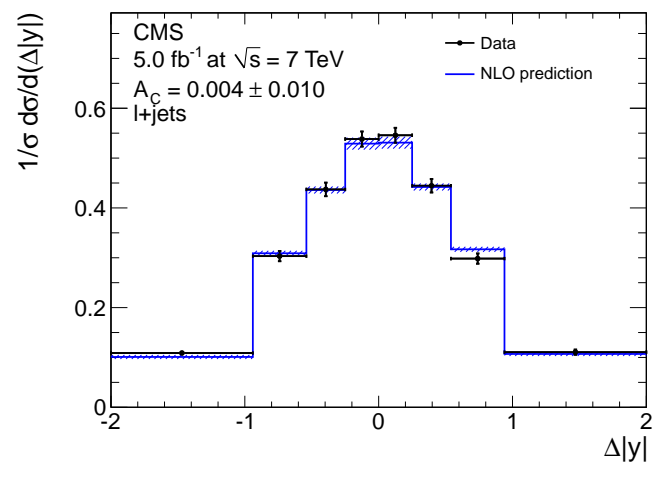

(a)

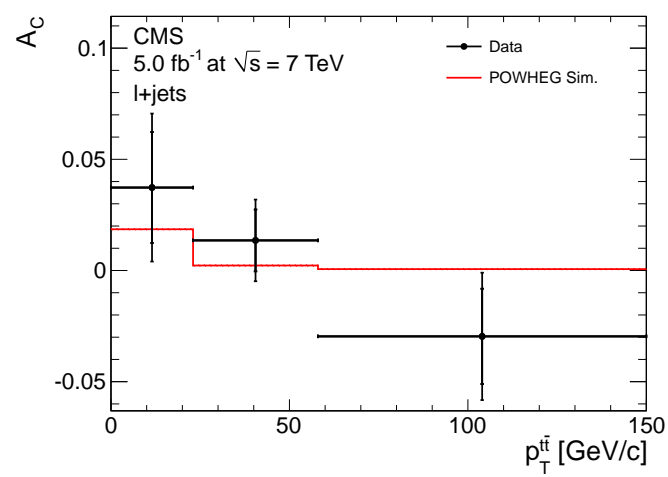

(c)

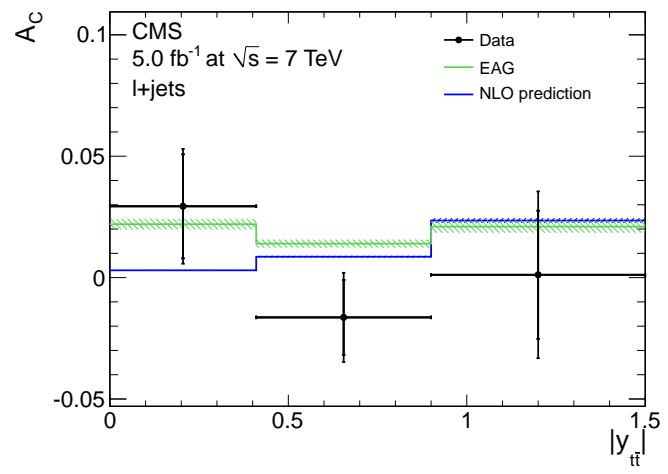

(b)

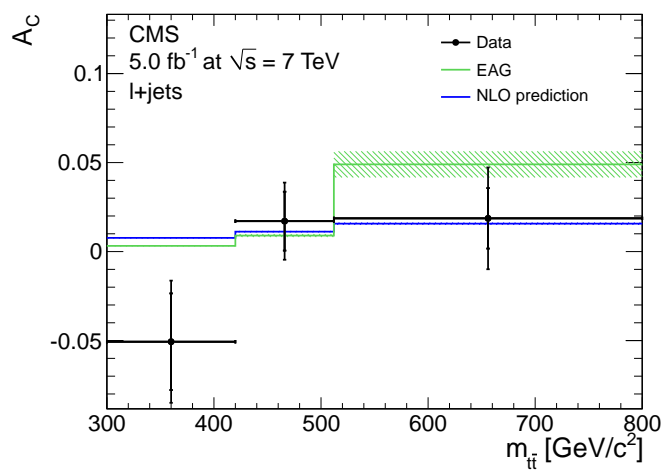

(d)

Figure 1. Background subtracted and unfolded inclusive $\Delta|y|$ distribution (a) together with differential $A_{C}$ measurements in bins of $\left|y_{t i t}\right|$ (b), $p_{T, t \bar{t}}(\mathrm{c})$ and $m_{t \bar{t}}(\mathrm{~d})$. The measurements are compared to SM NLO calculations and to the predictions of an effective model [9].

\section{$3 t \bar{t}$ spin correlation}

Due to the short lifetime of top quark, the $t \bar{t}$ spin correlation is propagated to the decay products. In dilepton final state, the charged leptons become correlated in the azimuthal angle $(\phi)$ in the laboratory frame. At $\sqrt{s}=7 \mathrm{TeV}$, CMS has measured the spin correlated $t \bar{t}$ content of data, $f$, using a template fit on $\left|\Delta \phi_{l^{+} l^{-}}\right|$distribution [13]. A binned likelihood fit to data is performed using three templates: one for backgrounds, one for SM $t \bar{t}$ and one for $t \bar{t}$ without spin correlation. The result, $f=0.74 \pm 0.08$ (stat.) \pm 0.24 (syst.), is consistent with the SM prediction, $f_{S M}=1$. The spin correlation coefficient in helicity basis, $A_{\text {hel }}$, is computed as $f \times A_{\text {hel }}^{S M}$ where $A_{\text {hel }}^{S M}(\mathrm{NLO})=0.31$ [14]. This leads to $A_{\text {hel }}=0.24 \pm 0.02$ (stat.) \pm 0.08 (syst.) which is compatible with $A_{\text {hel }}^{S M}$.

\section{The charge of top quark}

The SM predicts a charge of $+\frac{2}{3} e$ for the top quark. Using the dataset collected at $\sqrt{s}=7 \mathrm{TeV}$, CMS has tested the SM hypothesis against an exotic scenario with a top quark charge of $-\frac{4}{3} e$ [15]. The measurement is performed in muon+jets final state. It relies on the determination of b-quarks charge where the b-quark charge assignment is based on the charge of the soft muon from the electroweak B-meson decay. The top quark mass constraint is used to reconstruct the hadronic side of the event (where $\mathrm{W}$ decays hadronically) while the top quark charge is measured on the leptonic side. The normalized difference between the number of events with the SM and exotic top quark charge is taken as a test statistics, $A$. The measurement yields $A=$ $0.97 \pm 0.12$ (stat.) \pm 0.31 (syst.) which agrees with the SM $(A=1)$ and excludes the exotic scenario.

\section{$5 b$-quark content in top quark decay}

Top quark decays predominantly to a $b$-quark and a W-boson. Its coupling to other down-type quarks is highly suppressed in the SM. The measurement of $R=\frac{\mathcal{B}(t \rightarrow W b)}{\mathcal{B}(t \rightarrow W q)}$ is a test for this prediction of the SM while it is also an indirect measurement of $\left|V_{t b}\right|$ component of the CKM matrix. The CMS experiment has measured the $R$ ratio in the dilepton final state, using the $p p$ collisions at $\sqrt{s}=$ $8 \mathrm{TeV}$ [16]. The analysis is performed in 2-, 3- and 4-jets categories with different lepton flavors, $e e, e \mu$ and $\mu \mu$. The signal strength is obtained using a profile likelihood ratio with nuisance parameters to account for uncertainties. The correct assignment of the $b$-jet candidate to top quark is modeled with the invariant mass of the lepton and $b$-jet candidate, $m_{l j}$. The template for mis-assignments is taken from data. The $R$ ratio is measured using a profile likelihood with nuisance parameters based on the b-tagged jet multiplicity (up to 4 is allowed) in each category described above. The fit includes all possibilities for the (in)correct assignment of $b$-jet candidate to top quark with the $b$-jettop assignment probability from data. The b-tagging and 


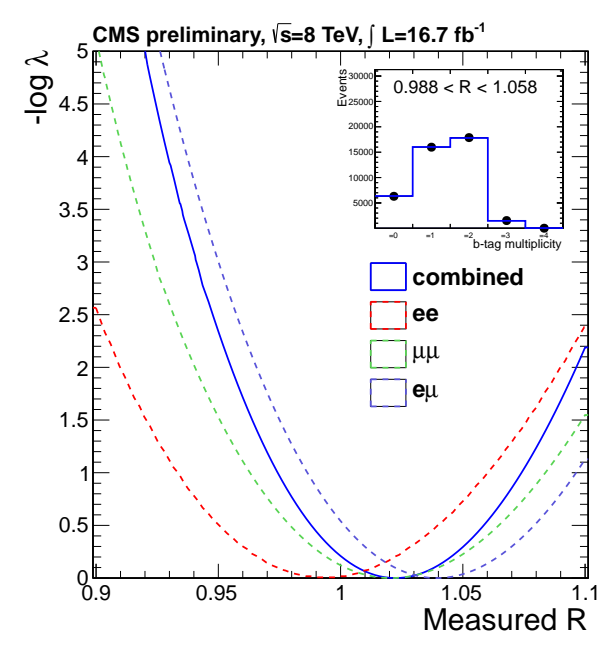

(a)

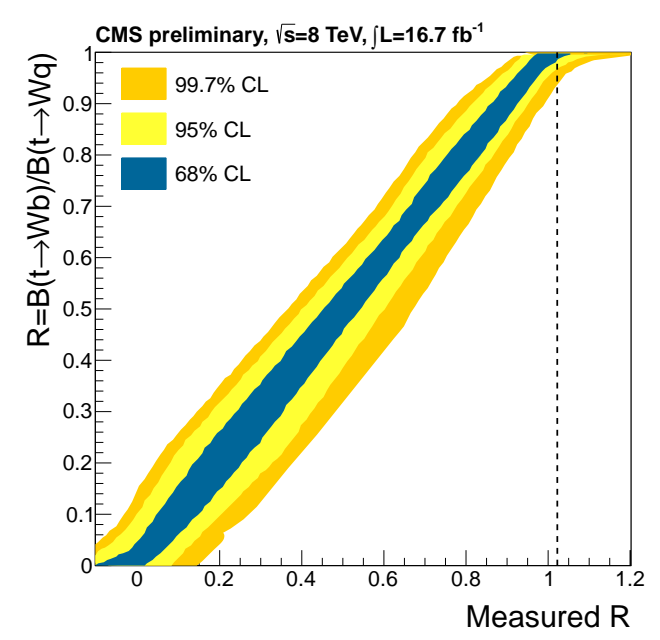

(b)

Figure 2. The profile likelihood in the exclusive dilepton channels (dashed lines) and in combination (solid line). The inset shows the inclusive $b$-tag multiplicity distribution and the result of the fit, (a). Limit bands at different C.L. on $R$, (b). For each true value of $R$ the range of allowed $R_{o b s}$ are shown at different C.L.

mistag efficiencies are calculated independently in a control data sample and are taken into account. The ratio is found to be $R=1.023_{-0.034}^{+0.036}$ (stat. + syst.), consistent with the SM expectations, $R_{S M}=0.998$. Assuming $R \leq 1$, a lower bound of $R>0.945$ is set at $95 \%$ C.L. using the Feldman-Cousins [17] approach. The profile likelihood for different lepton flavors and for the combination is shown in Figure 2(a) while Figure 2(b) illustrates the limit bands at different values of C.L.

The $R$ measurement leads to $\left|V_{t b}\right|=$ $1.011_{-0.017}^{+0.018}$ (stat. + syst.) with a lower bound of $\left|V_{t b}\right|>0.972$ for $V_{t b}<1$ at $95 \%$ C.L. This is the most precise measurement of $\left|V_{t b}\right|$ and the most stringent lower bound on this variable.

\section{Flavor Changing top quark decay to $Z q$}

The FCNC top quark decay of $t \rightarrow Z q$ is suppressed in the SM by the GIM mechanism. In some beyond SM theories [19] [18] however, it can be enhanced by $\sim 10$ orders of magnitude. At $8 \mathrm{TeV}$ center-of-mass energy, CMS has searched the rare process $t \bar{t} \rightarrow W b+Z q \rightarrow l v b+l l q$ using the entire data of 2012 [20]. In order to reduce backgrounds, the invariant mass of the $\mathrm{W}$ and $b$-jet candidate, $m_{W b}$, as well as the mass of the other top quark $\left(m_{Z j}\right)$ is required to be within $\sim 1.5 \sigma$ of the assumed top quark mass. The mass requirements are which was chosen without looking at the data. Figure 3 shows the the selected data points in the $\left(m_{W b} ; m_{Z j}\right)$ plane with the mass requirement indicated as a red box. Given the sample composition of diboson and DY (no $b$-jet), top FCNC (only one $b$-jet) and $t \bar{t}, t \bar{t} V$ and $t b Z$ (two $b$-jets), the $b$ tagging information is used to estimated background from data. Only one data event is observed after the full selection while the estimated number for background events is $3.14 \pm 4.97$ (stat.) \pm 0.17 (syst.). This results in an observed limit of $\mathcal{B}(t \rightarrow Z q)<0.07 \%$ at $95 \%$ C.L. which is the

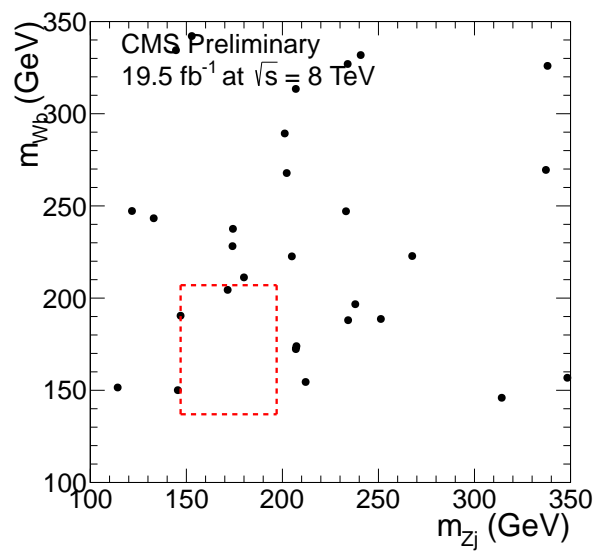

Figure 3. The $m_{W b}-m_{Z j}$ scattered plot for data events in FCNC $(t \rightarrow Z q)$ analysis. The red box indicates the top quark mass requirements.

most stringent limit. Compared to the CMS similar study at $\sqrt{s}=7 \mathrm{TeV}$ [21], the result has improved by a factor of 3 .

\section{Vector boson associated production}

The cross-section measurement of the vector boson associated top-pair production, $t \bar{t} W$ and $t \bar{t} Z$, is important to test the SM scenario for the $t-W$ and $t-Z$ couplings. Such processes are also irreducible backgrounds for $t \bar{t} H$. Using the entire $7 \mathrm{TeV}$ dataset, CMS has measured the $t \bar{t} Z$ crosssection in trilepton final state [22]. The same data is used to measure an inclusive $t \bar{t} V$ cross-section in same-sign dilepton events [22]. The measurement in dilepton analysis results in $\sigma_{t \bar{t} V}=0.43_{-0.15}^{+0.17}$ (stat.) $\pm_{-0.07}^{+0.09}$ (syst.) pb while in trilepton analysis, $\sigma_{t \bar{Z} Z}=0.28_{-0.14}^{+0.11}$ (stat.) $\pm_{-0.03}^{+0.06}$ (syst.) pb is 
obtained. Both measurements are consistent with the SM NLO calculations [23] [24].

\section{W-helicity fractions}

The helicity fractions of the W-boson from the top decay are sensitive to new physics in the $t W b$ vertex. They are defined as the partial width of top quark decay to a Wboson with left-handed $\left(F_{L}\right)$, right-handed $\left(F_{R}\right)$ and longitudinal $\left(F_{0}\right)$ polarization. The partial with of top quark can be expressed as a function of $\cos \theta_{l}^{*}$ where $\theta_{l}^{*}$ is defined in the top quark rest frame as the angle between the $\mathrm{W}$-boson 3-momenta and the 3-momenta of the down-type fermion in the W-boson rest frame. This distribution is exploited in CMS to measure the W-helicity fractions.

\subsection{Measurement in $t \bar{t}$ events}

The $p p$ collisions at $\sqrt{s}=7 \mathrm{TeV}$ are used for the measurement in muon+jets [25] and dilepton [26] $t \bar{t}$ final states. An event-by-event likelihood fit to data is performed where simulated signal events are reweighted. The weight function is defined as the ratio between the $\cos \theta_{l}^{*}$ distributions for an arbitrary set of helicity fractions in numerator and $F_{L}^{S M}, F_{0}^{S M}$ and $F_{R}^{S M}$ values in denominator. Two of the polarization fractions are extracted from the fit while the third is obtained using the unitarity condition, $F_{L}+F_{0}+F_{R}=1$. With $2.2 \mathrm{fb}^{-1}$ of data the measurement in muon+jets channel yields $F_{L}=0.393 \pm 0.045$ (stat.) \pm 0.029 (syst.), $F_{0}=0.567 \pm 0.074$ (stat.) \pm 0.047 (syst.) and $F_{R}=0.040 \pm 0.035$ (stat.) \pm 0.044 (syst.), in agreement with the SM predictions. This measurement is used to set limits on the real part of the anomalous $t W b$ couplings.

The dataset used for the dilepton channel amounts to an integrated luminosity of $4.6 \mathrm{fb}^{-1}$. The analysis results in $F_{L}=0.288 \pm 0.035$ (stat.) \pm 0.040 (syst.), $F_{0}=0.698 \pm$ 0.057 (stat.) \pm 0.063 (syst.) and $F_{R}=0.014 \pm 0.027$ (stat.) \pm 0.042 (syst.), with no deviation from the SM calculations.

\subsection{Measurement in single-top}

The CMS experiment has performed a similar measurement for the first time in single-top event topology with the muon+jets final state [27]. The reweighting method is employed in a binned likelihood fit to simultaneously measure the W-helicity fractions and the $\mathrm{W}+$ jets background contamination. The signal is defined as every process including top quark. The analysis is carried out at $\sqrt{s}=7 \mathrm{TeV}$ and $\sqrt{s}=8 \mathrm{TeV}$ and combined, yielding $F_{L}=0.293 \pm 0.069$ (stat.) \pm 0.030 (syst.), $F_{0}=0.713 \pm 0.114$ (stat.) \pm 0.023 (syst.) and
$F_{R}=-0.006 \pm 0.057$ (stat.) \pm 0.027 (syst.). The measured helicity fractions in single-top sector are compatible with the SM expectations, hence used to set limits on the real anoumalous $t W b$ couplings. The results are already comparable to those from $t \bar{t}$ despite the smaller statistics.

\section{Conclusion}

The CMS experiment has measured different properties of the top quark in production and decay. All CMS measurements of top quark properties are so far consistent with the Standard Model predictions.

\section{References}

[1] L. Evans and P. Bryant, JINST 3 S08001 (2008)

[2] The CMS Collaboration, JINST 3 S08004 (2008)

[3] J. H. Kühn and G. Rodrigo, Phys. Rev. Lett. 8149 (1998)

[4] J. H. Kühn and G. Rodrigo, Phys. Rev. D 59054017 (1999)

[5] CDF Collaboration, Phys. Rev. D 87092002 (2013)

[6] D0 Collaboration, Phys. Rev. D 84112005 (2011)

[7] CMS Collaboration, Phys. Lett. B 717129 (2012)

[8] J. H. Kühn and G. Rodrigo, JHEP 01063 (2012)

[9] E Gabrielli, Phys. Rev. D 84054017 (2011)

[10] CMS Collaboration, CMS-TOP-12-010 (2012)

[11] W. Bernreuther and Z.-G. SiPhys. Rev. D 86, 034026 (2012)

[12] S. Frixione, P. Nason and C. Oleari, JHEP 0711070 (2007)

[13] CMS Collaboration, CMS-TOP-12-004 (2012)

[14] W. Bernreuther, Z.-G. Si, Nuc. Phys. B 83790 (2010)

[15] CMS Collaboration, CMS-TOP-11-031 (2011)

[16] CMS Collaboration, CMS-TOP-12-035 (2012)

[17] G. Feldman and R. Cousins, Phys. Rev. D 573873 (1998)

[18] J. A. Aguilar-Saavedra, Phys. Lett. B 502115 (2001)

[19] G. Lu et al. Phys. Rev. D 68015002 (2003)

[20] CMS Collaboration, CMS-TOP-12-037 (2013)

[21] CMS Collaboration, CMS-TOP-12-004 (2012)

[22] CMS Collaboration, Phys. Rev. Lett. 110172002 (2013)

[23] J. Campbell and R. Ellis JHEP 07052 (2012)

[24] M. Garzelli et al. JHEP 11056 (2012)

[25] CMS Collaboration, CMS-TOP-11-020 (2011)

[26] CMS Collaboration, CMS-TOP-12-015 (2012)

[27] CMS Collaboration, CMS-TOP-12-020 (2012) 